\title{
Review
}

\section{Neurophysiological Mechanisms of Sleep-Dependent Memory Consolidation and Its Facilitation by Prenatal Choline Supplementation}

\author{
Ruey-Kuang Cheng, Christina L. Williams, and Warren H. Meck \\ Department of Psychology and Neuroscience \\ Duke University \\ Durham, NC 27708, USA
}

\begin{abstract}
Understanding the role of sleep in memory consolidation is an important topic in cognitive neuroscience because it could help us understand the functions of sleep as well as how memory is being consolidated off-line during sleep. Here we review recent studies that focus on the neurophysiological changes that occur during sleep and how these sleep-dependent changes could benefit memory consolidation. In particular, rapid eye movement (REM) sleep provides a preferential time window for memory consolidation across molecular, cellular and synaptic levels while slow-wave sleep (SWS) provides a second time window for memory consolidation at the neural-network level. It is proposed that high-frequency oscillations in the hippocampus (e.g., ripple events) serve as an endogenous trigger for long-term potentiation that plays a critical role in connecting a variety of sleep-dependent neurophysiological processes. We further suggest that repeated alternation of SWS and REM are needed in order to get a "good night's sleep". New findings from studies examining how prenatal choline supplementation produces organizational changes in hippocampal function, may provide a useful model for validating the importance of hippocampal ripple events in facilitating memory consolidation during both REM sleep and SWS. These data support our general conclusion that the types of neural plasticity supported by prenatal choline supplementation and sleep are vital for cognitive health, because they produce a host of neurophysiological changes, some of which are also important for memory consolidation.
\end{abstract}

Key Words: neuronal oscillations, immediate early gene expression, long-term potentiation, neurogenesis, Pattern replay, gamma oscillations, sharp-wave ripple oscillations, cortex, hippocampus, nutrition

\section{Introduction}

Sleep is a basic function needed for survival and is a physiological state that is widely shared by all members of the animal kingdom. Although the amount of sleep differs among species, it even differs as a function of age among individuals within the same species (for a comparative review, see 122). On average, adult humans sleep $8 \mathrm{~h}$ a day. Because one third of our lifespan is spent asleep, understanding the functions of sleep (e.g., in terms of homeostatic and cognitive functions) has long been an intriguing topic for philosophers, physicians, and especially for neuroscientists. Modern sleep research began with the seminal report by Aserinsky and Kleitman (2 and 37). In essence, Kleitman and colleagues were the first to characterize different stages of sleep by utilizing electroencephalography (EEG) in human

Corresponding author: Dr. Warren H. Meck, Department of Psychology and Neuroscience, Genome Sciences Research Building II - $3^{\text {rd }}$ Floor, 572 Research Drive - Box 91050, Duke University, Durham, NC 27708, USA. Phone: (919) 660-5765, Fax: (919) 660-5798, E-mail: meck@psych.duke.edu

Received: December 4, 2008; Revised: May 23, 2009; Accepted: June 5, 2009.

(C)2009 by The Chinese Physiological Society. ISSN : 0304-4920. http://www.cps.org.tw 
Table 1. Distinct oscillatory patterns in hippocampal and cortical regions during different sleep states

\begin{tabular}{lll}
\hline & \multicolumn{1}{c}{ NREM Sleep } & \multicolumn{1}{c}{ REM Sleep } \\
\hline $\begin{array}{l}\text { Surface EEG in the cortex } \\
\text { from human studies }\end{array}$ & $\begin{array}{l}\text { Slow oscillations ranging from 1-4 Hz } \\
\text { (delta) and from 7-14 Hz (theta) (ref. 121). }\end{array}$ & Fast (20-50 Hz) oscillations (ref. 131). \\
$\begin{array}{l}\text { LFP in the hippocampus } \\
\text { from animal studies }\end{array}$ & $\begin{array}{l}\text { Large-amplitude irregular activity (LIA) } \\
\text { with some fast sharp-wave oscillations } \\
(\sim 200 \mathrm{~Hz})(\text { ref. 61). }\end{array}$ & $\begin{array}{l}\text { High power of theta oscillations (5-7 Hz) } \\
\text { (ref. 32). }\end{array}$ \\
\hline
\end{tabular}

NREM = Non-rapid-eye movement sleep; REM = rapid eye movement sleep; EEG = Electroencephalography; LFP = local field potentials

participants, thus beginning an era in which sleep was studied not only quantitatively (i.e., how long we sleep), but also qualitatively (i.e., how well we sleep). As a result of this major advance, sleep is no longer considered to be a state of quiescence. Instead, we now know that dynamic and complex EEG patterns occur repeatedly across a period of sleep and the functions of sleep (or more importantly, the cognitive functions of sleep) have become accessible to neuroscientists. In the current article, we review critical sleep studies with a special focus on the hypothesized roles of sleep in memory consolidation.

\section{Stages of Sleep}

In human sleep studies that utilize brain EEG and electrooculography (EOG), sleep can be classified into two distinctive states-non-rapid eye movement (NREM) sleep and rapid eye movement (REM) sleep. NREM sleep can be further partitioned into 4 different stages with Stages 1 and 2 characterized by sleep spindles and K-complexes while Stages 3 and 4 are characterized by slow waves (also known as slowwave sleep, SWS). During early development, human infants spend about $50 \%$ of their total sleep time in REM sleep and this amount gradually declines to 20\%-25\% over the first two years of life (18). Both REM and NREM sleep states cycle alternately during a full night of sleep, usually 4 cycles per night's sleep in healthy adult humans (36). From the EEG recording, REM sleep is characterized by patterns of short-scale and fast $(20-50 \mathrm{~Hz})$ oscillations in the cortex, a pattern known as cortical "synchronization at high-frequency" during REM sleep (e.g., see 131, 132). During SWS, however, the surface EEG shows slow-wave oscillations ranging from 1-4 Hz (delta) and from 7-14 Hz (spindle) that are typical patterns for this sleep state $(42,44,101)$.

In contrast to studies of human sleep patterns that record EEG's via surface electrodes, sleep experiments in cats, rodents, and other animals, typically involve the implantation of recording electrodes directly into the brain (but see 51 and 115 for exceptions). Electrophysiological recordings from the hippocampus are commonly reported in the animal literature because most of these studies examine spatial behaviors that rely on the hippocampus-although not all types of learning and memory are equally dependent on this brain region $(71,79,89,94,105)$. In general, SWS and REM sleep in animals can be determined by specific oscillatory patterns of the hippocampal local field potentials (LFP). For example, during REM sleep, the hippocampal LFP exhibits strong theta oscillations, a frequency band that is commonly defined in the range of 5-12 Hz (32). During SWS sleep, the hippocampal LFP displays slow oscillations (in delta band that ranges from 1-5 Hz, see 133-135) occasionally mixed with fast oscillations (up to 200 $\mathrm{Hz}$ ) as described by Jarosiewicz et al. (61). It is also possible that sleep studies can be conducted by recording neural signals from regions other than the hippocampus, such as the striatum (75). In conclusion, different stages of sleep are characterized by distinct neural oscillatory patterns in both the surface EEG and in the hippocampal LFP. It is these distinct oscillatory patterns that allow researchers to classify each sleep stage and investigate how each sleep stage relates to learning and memory (see Table 1). In the following sections, we will discuss neurophysiological changes that occur in each sleep stage and their potential role in memory consolidation.

\section{Characteristics of REM Sleep Relevant to Memory Consolidation}

When determining any potential functions of REM sleep, one must keep in mind that human infants spend half of their sleep time in the REM sleep stage (18), an amount that is much greater than the amount reported for human adults. Hence, whatever the functions of REM sleep are, it is either that infant REM sleep is much less efficient than adult REM sleep or that the infant's REM sleep serves a different function compared to the adult's REM sleep. That is, some of the functions of REM sleep could change as people age whereas other functions might be shared across the lifespan. While it remains to be determined which functions of REM sleep belong to the first category 
(i.e., change as a function of age) and which functions belong to the second category (i.e., constant as a function of age), we propose that the following 3 functions fall into the second category and relate to memory consolidation: Immediate early gene expression (IEG), maintenance of long-term potentiation (LTP), and neurogenesis.

\section{Immediate Early Gene Expression and Long-Term Potentiation during REM Sleep}

The induction of IEG has been viewed as an early indicator of brain region activation that leads to further molecular and cellular processing and ultimately protein synthesis. Hence, one can speculate that as memory is being consolidated, there must be activation of gene expression before any new protein is synthesized in support of synaptic growth (66). Indeed, after exposing rats to an enriched environment, an increase of zif-268 gene expression during REM sleep, but not SWS, is observed in the hippocampus (113). Furthermore, inducing LTP in the hippocampus when rats are awake also triggers zif-268 gene expression in extrahippocampal regions during subsequent REM sleep (113). More importantly, deactivating the hippocampus during REM sleep abolishes the propagation of zif-268 gene expression in extrahippocampal regions, further suggesting that REM sleep provides an exclusive time window for the expression of zif-268 following LTP induction (114).

The protocol for inducing the expression of zif-268 in the hippocampus is similar to the protocol for inducing hippocampal LTP. For example, both zif-268 expression and LTP can be induced by highfrequency stimulation and can be blocked by NMDA receptor antagonists (31). This suggests that these two phenomena share similar neural mechanisms. Because of this similarity, if zif-268 expression is related to REM sleep, one can also assume that hippocampal LTP is also related to REM sleep. Indeed, recent evidence has found that REM sleep deprivation, but not SWS deprivation, impairs the maintenance of hippocampal LTP $(17,60,72)$. The devastating effect of REM sleep deprivation on LTP may be able to explain the memory deficits observed in sleep deprived rats tested in a variety of hippocampaldependent tasks (e.g., 34, 35, 52, 77, 126, 127-but see 112 for results from a human study). In summary, REM sleep appears to provide a time window for the induction of IEGs, such as zif-268 and the maintenance of LTP, both of which are important for memory consolidation and sensitive to REM sleep deprivation.

\section{Neurogenesis during REM Sleep}

At the cellular level, recent studies have found

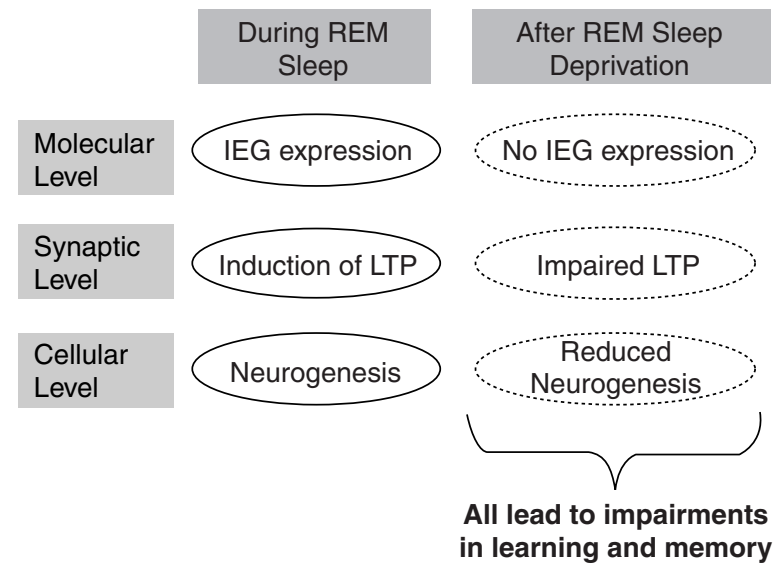

Fig. 1. Rapid eye movement (REM) sleep-dependent neurophysiological changes. REM sleep provides a time window for a) immediate early gene expression (e.g., zif-268) at the molecular level, for b) maintenance of long-term potentiation (LTP) at the synaptic level, and for c) neurogenesis at the cellular level. REM sleep deprivation likely impairs all three neurophysiological changes and leads to impaired memory performance.

that total sleep deprivation is deleterious to adult neurogenesis in the dentate gyrus of the hippocampus $(54,55)$. By selectively depriving rats of different stages of sleep, researchers are able to demonstrate that the deprivation of REM sleep, but not of SWS, is responsible for the reduction in neurogenesis (53). Recent evidence has suggested that adult neurogenesis in the hippocampus is involved in certain types of learning and memory (e.g., see 12, 48, 67, 141 or 120 for a recent review). Hence, regulation of hippocampal neurogenesis by REM sleep may provide a potential connection between sleep and memory consolidation. Indeed, an increase of hippocampal neurogenesis is observed only in rats that were trained in a hippocampal-dependent water-maze task (56). Furthermore, sleep restriction during the first $6 \mathrm{~h}$ after the initial training abolished this elevated neurogenesis as well as impaired the behavioral performance of rats in subsequent testing (56). As a result, REM sleep, especially the cycles occurring during the first $6 \mathrm{~h}$ following initial training may provide a time window for hippocampal neurogenesis that is critical for learning and memory. Interestingly, inducing LTP in the hippocampus by high-frequency stimulation can also enhance hippocampal neurogenesis while blocking NMDA receptors can abolish both LTP induction and neurogenesis in the hippocampus $(12,29)$.

In conclusion, the evidence outlined above connects REM sleep with hippocampal IEG expression, LTP maintenance, and neurogenesis as illustrated in Fig. 1. Connecting REM sleep to these neurophysiological changes provides the basis for 
understanding the role of REM sleep in memory consolidation, which is consistent with the major findings from numerous studies $(9,32-34,102,128-129)$.

\section{Characteristics of SWS Relevant to Memory Consolidation}

In contrast to the long-held interest in studying the connections between REM sleep and memory consolidation, understanding the role of SWS in memory consolidation has now gained momentum in recent years sparked by series of reports that unveiled the complex neural-network interactions and highfrequency oscillations in the hippocampus during SWS (e.g., 45, 65).

\section{Pattern Replay in the Hippocampus during SWS}

If the process of memory consolidation could occur during sleep states after initial encoding during waking states, then one would expect that the consolidation process would recruit the same neural connections and/or replay the same patterns of neural activity that were originally involved in the representation of the information. It is the neural representation of information that is being consolidated from a relatively fragile, short-term form into a more stable, long-term form. Consequently, the same subset of neurons that were originally involved in processing and representing the "to be remembered" sensory input should be activated again when the information is transferred from recent memory into remote memory (43). The phenomenon of the same group of neurons being activated again during sleep is called "pattern replay" and was first reported by Wilson and McNaughton (148). Specifically, rats' hippocampal place cells that fired together during a spatial task tended to fire together again during subsequent SWS. Later experiments replicated this finding (124), but did not find similar pattern replay during REM sleep (68, but see 73). Hence, neuronal pattern replay during SWS seems to be an underpinning of memory consolidation. In addition, Lee and Wilson (70) showed that neural pattern replay during sleep occurs about 20 times faster than the original pattern took to unfold in real time. That is, the firing pattern is preserved, but the time scale for the replayed pattern is compressed approximately 20 -fold. This pattern replay phenomenon and its compressed time scale have also been observed by Ji and Wilson (62), further suggesting that SWS provides a time window for replaying the neural response patterns that may reflect memory consolidation for certain types of memory $(109,130,138,143)$. Indeed, slow-wave oscillations are thought to be associated with synchrony between cortical regions that provide an environment for large-scale coordinated information transfer during SWS $(15,39,57,118,140,149)$. In this regard, Buzsaki (14) has proposed that cooperative neuronal activity patterns during SWS may reflect the transfer of information in "quantal units" from the hippocampus to the prefrontal cortex where they are consolidated into more stable representations with overlapping and distributed connections (also see $68)$.

\section{High-Frequency Oscillations in the Hippocampus during SWS}

As noted above, a paradoxical characteristic of slow wave sleep is that the hippocampus actually displays some high-frequency oscillations (up to 250 $\mathrm{Hz}$ ) during this sleep stage (13). These high frequency oscillations may occur because of the physical property that allows wave forms with different frequency bands to be embedded with each other, i.e., high frequency waves can reside in slow frequency waves. Specifically, the high-frequency oscillations that occur during SWS are comprised of ripple oscillations with a range from 100-250 Hz. In order to focus on these ripple oscillations, it is a common practice to use digital filters to isolate the ripple component from the raw oscillatory traces with full spectrum waveforms (for details about digital filters, please see 74). In the filtered LFP traces, there is another distinctive feature referred to as a ripple event, which is defined based on a transient magnitude increase over a short period of time. Once individual ripple events are identified, one can quantify many aspects of these events as parameters - the frequency of their occurrence in a period of time, their mean amplitude, and their mean duration or width as illustrated in Fig. 2. Although hippocampal ripple events can occur during the waking state $(3,30,69)$, numerous studies report that during sleep ripple events preferentially occur during SWS, and are less frequent during REM sleep in rodents $(68,99)$, monkeys $(125)$, and humans $(3$, 69, 98).

What impact, if any, do hippocampal ripple events during SWS have on memory consolidation? As mentioned above, pattern replay is a feature of neural activity during SWS and the general consensus is that pattern replay may be involved in the interaction between hippocampal and neocortical regions $(64-65,145)$. During this replay process, researchers have found that hippocampal ripples are the main identifiable feature of cortico-hippocampal interactions during SWS (e.g., 7, 121, 123). In a more detailed analysis, the replay process is "chunked" in separate frames and each frame is characterized by a burst of hippocampal ripple events reflecting information transfer into long-term memory at a 


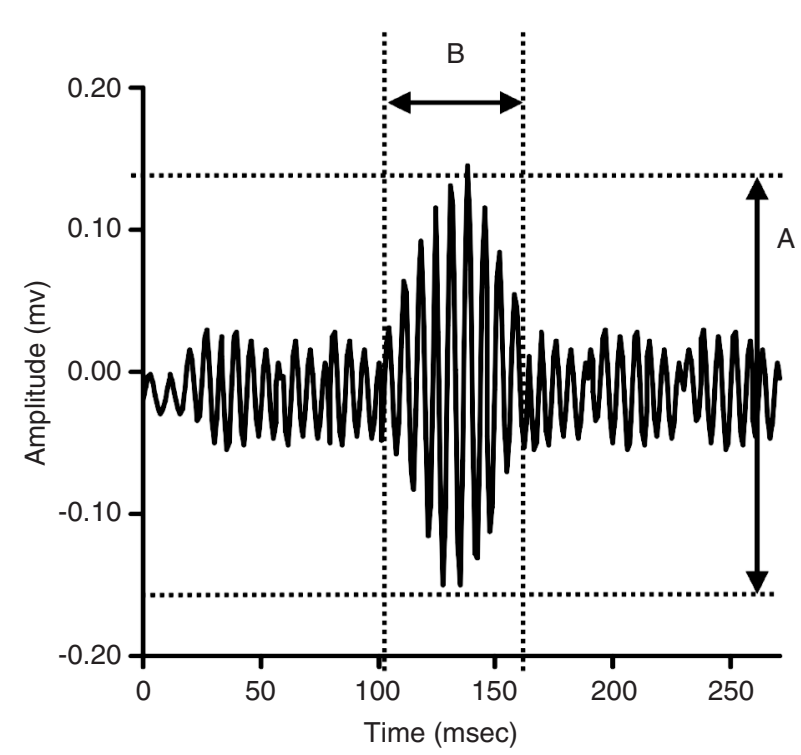

Fig. 2. A representative hippocampal ripple event. The amplitude of this ripple event is about $32 \mathrm{mv}$ as indicated by the distance (A) while the duration (or width) of this ripple event is about $60 \mathrm{~ms}(\mathrm{~B})$.

particular baud rate $(10,62,81,83,107,109)$. Taken together, the process of memory consolidation during SWS can be characterized by the following scenario: A group of hippocampal neurons that showed correlated firing during the acquisition of information in the waking state also show correlated firing, but in a time compressed manner (i.e., pattern replay) during SWS, a time period during which ripple events also preferentially occur.

To further establish the causal relationship between the features of SWS (i.e., pattern replay and ripple events) and memory consolidation, one might change the pattern replay and observe whether memory consolidation is affected. While changing the pattern replay is theoretically possible, it is extremely difficult to identify all of the neurons that are responsible for the representation of a specific external event and manipulate them in an appropriate manner. Consequently, measuring and manipulating ripple events is considered to be more plausible experimentally. Indeed, recent evidence suggests that hippocampal ripple events are involved in the storage of new spatial information (27), and also serve as an indicator of the initial learning experience during waking periods (108). During subsequent SWS, the magnitude, duration, and frequency of ripple events are all increased during the first hour of sleep following the initial learning experience (40). Furthermore, if these ripple events are disrupted during SWS, the acquisition of a spatial task is impaired (47). In addition to these rodent studies, the importance of SWS in memory consolidation has also been reported in human par-

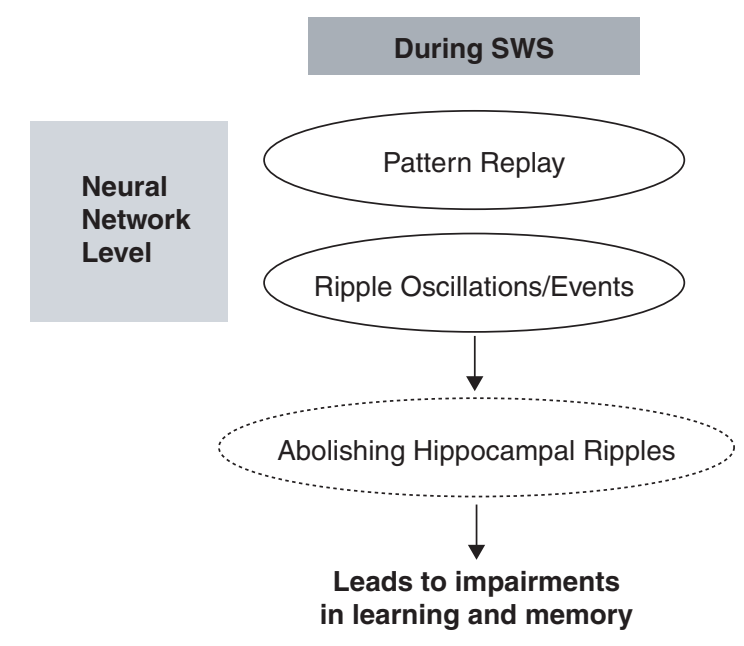

Fig. 3. Slow-wave sleep (SWS) dependent neurophysiological changes. Pattern replay is the process of having the same group of neurons activated again during sleep that were originally activated by a waking experience. This process is also accompanied by hippocampal ripple events. If these ripple event are disrupted, subjects exhibit impairments in learning and memory (see 47).

ticipants $(45,78,98)$. In particular, the co-occurrence of hippocampal ripple events together with cortical slow-wave activity (e.g., spindles and delta oscillations) during SWS may provide a bridge mechanism for further memory processing between these two brain regions $(30,57,70)$. In conclusion, the importance of SWS in memory consolidation is proposed to be at the neural-network level as its two main neurophysiological features (e.g., pattern replay and ripple oscillations/events - as diagrammed in Fig. 3 ) are directly related to hippocampal - neocortical interactions.

\section{Alternation of Sleep Stages: Possible Benefits for Memory Consolidation?}

Perhaps because most sleep studies have focused on the role of individual sleep stages in memory consolidation (i.e., SWS or REM sleep, but not both), it is still not understood why a "good night's sleep" is composed of multiple episodes of SWS and REM sleep that alternate several times. It is also unclear whether this cycling of sleep stages provides any benefit to memory consolidation. In general, a good balance between the amount of SWS and REM sleep seems to be important because many psychological disorders, especially depression (106), are more or less related to an imbalance between these two sleep stages (38, 142). To account for this unexplored issue, we propose a novel theoretical framework regarding how the alternation of sleep stages may provide a global neural mechanism for memory consolidation. 


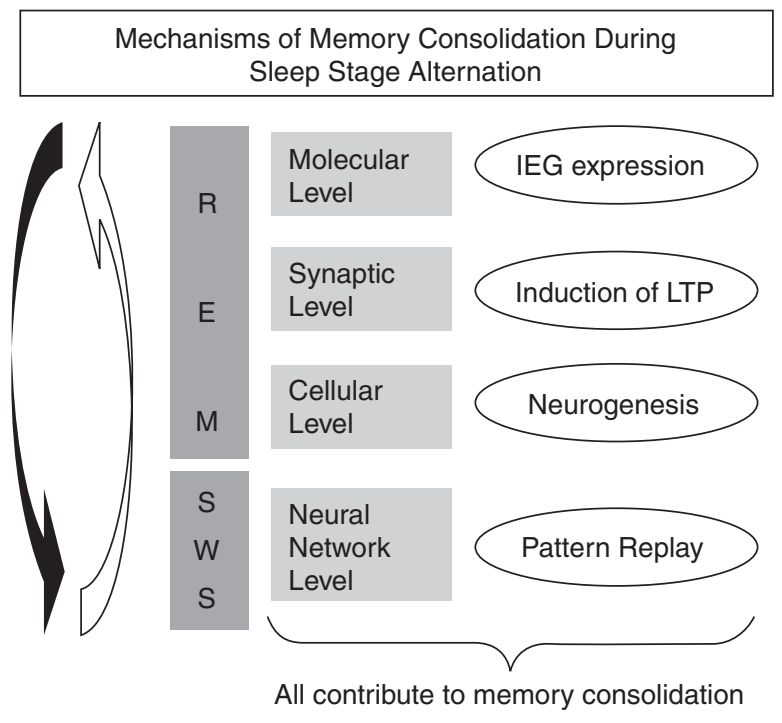

Fig. 4. A global neural mechanism for sleep-dependent memory consolidation that includes all the neurophysiological changes proposed to occur during each sleep stage.

As reviewed in previous sections, REM sleep provides a preferential time window for IEG expression, maintenance of LTP, and neurogenesis in the hippocampus from the molecular to the synaptic and cellular levels. In contrast, SWS provides a preferential time window for pattern replay and ripple oscillations/events at the neural-network level. When combining the two sleep stages, it vertically integrates several neural mechanisms from the molecular level to the neural-network level involved in memory consolidation as illustrated in Fig. 4. In other words, if one considers memory consolidation to ultimately result in the modification of synaptic weights within a distributed cortical network, then the specific hippocampal-neocortical interactions occurring during SWS and REM sleep serve to "set the stage" for this information transfer. The idea that memory consolidation is "a continuing series of biological adjustments" fits well with the characteristics of sleep stage alternation (136). To further delineate the mechanisms of those biological adjustments and how they integrate "vertically", we propose that hippocampal ripple events are playing a critical role in the process of memory consolidation during sleep for the following reasons.

First, hippocampal ripple events may serve as the in vivo mechanism for the induction of LTP because their frequency range overlaps with the operational definition of the high-frequency stimulation required to induce hippocampal LTP in vitro. In fact, a single burst of electrical stimulation at $100 \mathrm{~Hz}$ given at the peak phase of theta oscillations in the hippocampal slice can induce robust LTP, while the same burst of stimulation given at the trough of theta

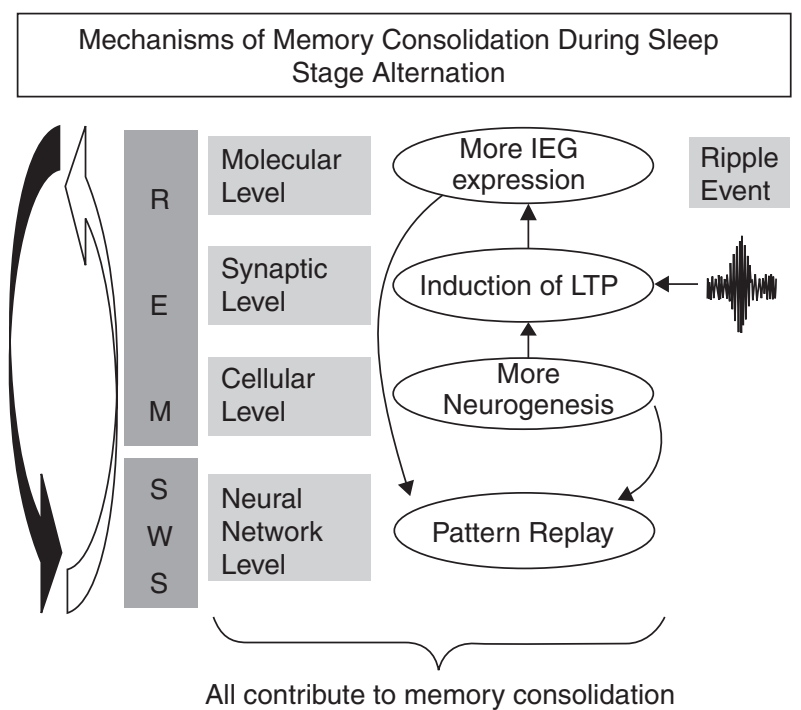

Fig. 5. Hippocampal ripple events play a vital role in this neural mechanism because of its ability to induce long-term potentiation (LTP). This in vivo-induced LTP during rapid eye movement (REM) sleep initiates gene expression and neurogenesis, all providing the "raw materials" for further processing during slow-wave sleep (SWS).

oscillations induces long-term depression $(58,104)$. As a consequence, this bidirectional synaptic plasticity, regulated by the synchronization of highfrequency stimulation with hippocampal theta oscillations, enables the modification of synaptic weights by either strengthening or weakening the connections (59). The idea that in vivo high-frequency oscillations could serve this role has previously been suggested $(6,14,23)$ and is supported by empirical evidence (8). Second, this in vivo-induced LTP further triggers IEG expression (114) in route to protein synthesis at the molecular level as well as stimulating neurogenesis at the cellular level during REM sleep $(12,29)$. Finally, to incorporate the newly-synthesized proteins and newly-generated neurons into established neural networks, pattern replay is recruited during SWS such that these neural networks go through a process of reorganization and reactivation that ultimately consolidates the encoded information through NMDA-dependent consolidation processes as illustrated in Fig. 5. Once the NMDA receptor is activated, it triggers the calcium-signaling cascades inside the neurons that eventually lead to modification of synaptic weights (137), and growth of dendrites (150); both proposed as key elements in models for long-term memory (e.g., 28, 119). Therefore, we assume that a "LTP $\rightarrow$ gene expression $\rightarrow$ neurogenesis" process provides the necessary materials to support consolidation and storage of information in longterm memory. In this manner, the benefit of alternating sleep stages begins during SWS with high-frequency 
hippocampal ripples that induce LTP thereby triggering a sequence of molecular and cellular events during pattern replay.

Based on the theoretical framework described above, one can speculate that the expression of memory-related genes and neurogenesis during REM sleep, both triggered by in vivo high-frequency stimulation are providing the active ingredients for further information processing in subsequent stages of SWS. This provides one explanation as to why sleep stages alternate - each sleep stage generates products for further processing in the subsequent sleep stage. The fact that human infants spend half of their sleep time in REM sleep supports this proposal because developing infants require more "raw materials" for developing neural connections (i.e., more synaptic proteins and neurogenesis). As most researchers agree that memory consolidation is an ongoing process requiring gene expression and protein synthesis ( 66 - but see ref. 50 for an alternative view), the repeated alternation of REM sleep and SWS is actually beneficial to the ongoing process of memory consolidation. In other words, this process requires all the neurophysiological changes that occur during sleep with some preferentially occurring during REM sleep and others during SWS. These molecular and cellular processes function optimally only if the two sleep stages alternate in time. If any of these neurophysiological changes are disrupted (e.g., by sleep deprivation), the net result is compromised. While it is likely that the alternation of sleep stages benefits memory consolidation, one would expect that the consolidation process will not be fully completed in just one sleep cycle or even in one night's sleep. Because memory consolidation is likely to be an ongoing process (136), one might expect that adjustments occur over different time scales (e.g., hours, days, or weeks) and at different levels (e.g., molecular, cellular, and neural-network). For example, when new proteins are produced after gene expression near the nucleus of the cell body, it takes time for these proteins to be transported to synapses where the synaptic weights are being modified. Accordingly, it is likely that sleep, especially the alternation of sleep stages across several nights, may be crucial to this ongoing process.

Even within a sleep state, multiple oscillatory events may be critical to memory formation. For example, one physical property of waves is that waveforms with different frequencies can "add on" to each other thereby constituting a complex waveform. Hence, it is tempting to speculate that hippocampal ripple events within the frequency range of 100-250 Hz, may be the key to "unlock" specific gene expression that is critical to memory consolidation. For example, ripple events in range of 150-180
$\mathrm{Hz}$ could preferentially induce the expression of zif-268, while ripple events in a different frequency range (e.g., 180-200 Hz) could induce the expression of other IEGs. Thus, a full spectrum of high-frequency oscillations may trigger a family of IEGs. Although this proposal remains to be empirically tested, it provides insight into the complex nature of the relationship between neuronal oscillations in sleep and memory consolidation.

Finally, does memory consolidation exclusively occur during sleep states, but not during the awake state, which is the traditional position taken on sleep and memory consolidation? Not necessarily, if the proposal outlined above is correct, one would expect that a subject would need to enter a sleep state soon after the initial exposure to a new experience in order to consolidate it into long-term memory. Recently, a growing body of evidence suggests that some aspects of memory consolidation begin as early as the first rest state following the initial encoding $(3,5-6,116)$. An overview of this alternative theory of sleep and memory consolidation has recently been presented by Axmacher and colleagues (4). Within the sleep literature, one can also find that some memory tasks are relatively immune to REM sleep deprivation while others are immune to SWS deprivation. At first glance, this may violate the prediction of our "vertically-integrated" model of memory consolidation in which both sleep stages cooperate. It should be noted, however, that different types of memory may require differential levels of consolidation during SWS and REM sleep (e.g., 116, 142). That is, some types of memory may require more REM sleep-related consolidation processes while others may require more SWS-related consolidation. The issue becomes even more relevant when one takes into account the interactive theory of sleep and memory consolidation (4). For example, some episodic memory tasks do not heavily rely on SWS-related memory consolidation and the storage process can be achieved during waking states shortly after initial encoding. This will make this type of task appear independent of SWS and immune to selective SWS deprivation. It is beyond the scope of the current review to provide all of the details distinguishing different types of memory from their dependency on different sleep stages. Instead, our focus is on the overall contribution of each sleep stage and the benefit of their sequential alternation to memory consolidation, especially in the case where hippocampal ripple events are enhanced by prenatal choline supplementation.

\section{Prenatal Choline Availability and Sleep-Dependent Memory Consolidation}

Dietary choline supplementation during preg- 
nancy, especially during embryonic days $12-17$ in rats, has been shown to produce long-term facilitative effects on cognition in adult offspring $(86,93)$. Specifically, adult rats treated prenatally with choline supplementation exhibit enhanced memory capacity and precision of both temporal and spatial memory (25-26, 82, 84-88, 91-92, 97). These beneficial effects in prenatally choline supplemented (SUP) rats last throughout adulthood and protect against age-related declines in memory compared to rats that received sufficient (CON) or deficient (DEF) amounts of choline prenatally $(21-22,86,93)$.

In addition to the behavioral studies outlined above, recent neurobiological studies have demonstrated that prenatal choline supplementation indeed causes long-term neurophysiological adaptations in the hippocampus $(71,100)$. For example, choline metabolism in the hippocampus in adult rats is sensitive to their prenatal choline availability (1920). Furthermore, prenatal choline supplementation also increases nerve growth factor (NGF), brainderived nerve growth factor (BDNF), insulin growthlike factor 1 (IGF-1), vascular endothelial growth factor (VEGF), a family of proteins that are critical for survival and maintenance of neurons in the hippocampus of adult rats $(48-49,117,152)$, and elevates basal hippocampal neurogenesis $(48-49,151)$. Increased levels of these growth factors and neurogenesis are considered to be facilitative of certain types of learning and memory $(1,16,41)$. In terms of neuroplasticity, prenatal choline supplementation has also been shown to attenuate the neuropathological response to status epilepticus in the adult rat hippocampus (152) and hippocampal slices from adult rats given prenatal choline supplementation exhibit a lower threshold for the induction of long-term potentiation $(63,110)$. Consequently, it is of particular interest to understand how hippocampal oscillations and sleep patterns in adult rats differ as a function of prenatal choline availability. Indeed, we recently reported that the power of gamma band oscillations and the amplitude of hippocampal ripple events in 1214 mo old rats given prenatal choline supplementation is higher compared to CON and DEF rats during SWS and REM sleep $(23,24)$. In addition, the SUP rats also exhibited more REM sleep episodes that contain hippocampal ripple events compared to CON and DEF rats (24) further indicating that the quality of sleep (e.g., hippocampal ripple events occurring during REM sleep) is sensitive to prenatal choline availability in male and female rats. Taken together, these data implicate an ontogenetic mechanism for regulating memory capacity and precision that is expressed by sleep-dependent memory consolidation processes (for review, see 93).

As described in the previous sections, hippoc- ampal ripple events are purported to play a crucial role in sleep-dependent memory consolidation. The key to this process is in the ability of high-frequency ripple oscillations to induce LTP in the hippocampus $(6,8)$ which in turn up-regulates gene expression (114) and neurogenesis (29). Although the precise patterns of gene expression and their functions following prenatal choline exposure remain to be determined $(95,96)$, we can infer a role in memory consolidation based on the finding that NGF and BDNF are up-regulated by prenatal choline supplementation $(48,117,151)$. This up-regulation of growth factors, in turn, increases neurogenesis and synapse formation and ultimately contributes to the enhancement memory function (41). These findings support our proposal that more "raw materials" are being generated during REM sleep due to the epigenetic effects of prenatal choline supplementation $(11,80,144,146,153,154)$ and therefore facilitate neuronal pattern replay and information transfer during subsequent SWS as illustrated in Fig. 6. Given that prenatal choline supplementation has been found to elevate the amplitude of hippocampal ripple events and increases their occurrence during REM sleep in adult rats (24), it is tempting to speculate that the long-term impact of prenatal choline availability on memory function can be explained, in part, by alterations in the generators of hippocampal ripple events $(76,103)$. In the case of prenatal choline supplementation, increased excitability of hippocampal ripple generators may be associated with changes in GABAergic and glutamatergic synapses on CA1 pyramidal neurons $(111,139)$ as well as increases in the size of cholinergic cell bodies in the basal forebrain (147).

\section{Conclusion}

While the growing consensus is that sleep provides a preferential time window for memory consolidation, it remains to be determined how each sleep stage cooperatively contributes to memory consolidation and why the sleep stages alternate repeatedly during a night of sleep. For example, why didn't sleep evolve such that REM sleep occurs during the first half of the night and SWS during the second half or vice versa? Here, we propose a theoretical account for the alternation between REM sleep and SWS such that each neurophysiological change in each sleep stage cooperatively participates in the process of memory consolidation during sleep. Specifically, neurophysiological changes during REM sleep seem to provide "raw materials" at molecular level (gene expression), synaptic level (LTP), and cellular level (neurogenesis). These "raw materials" then become the building blocks for consolidating 


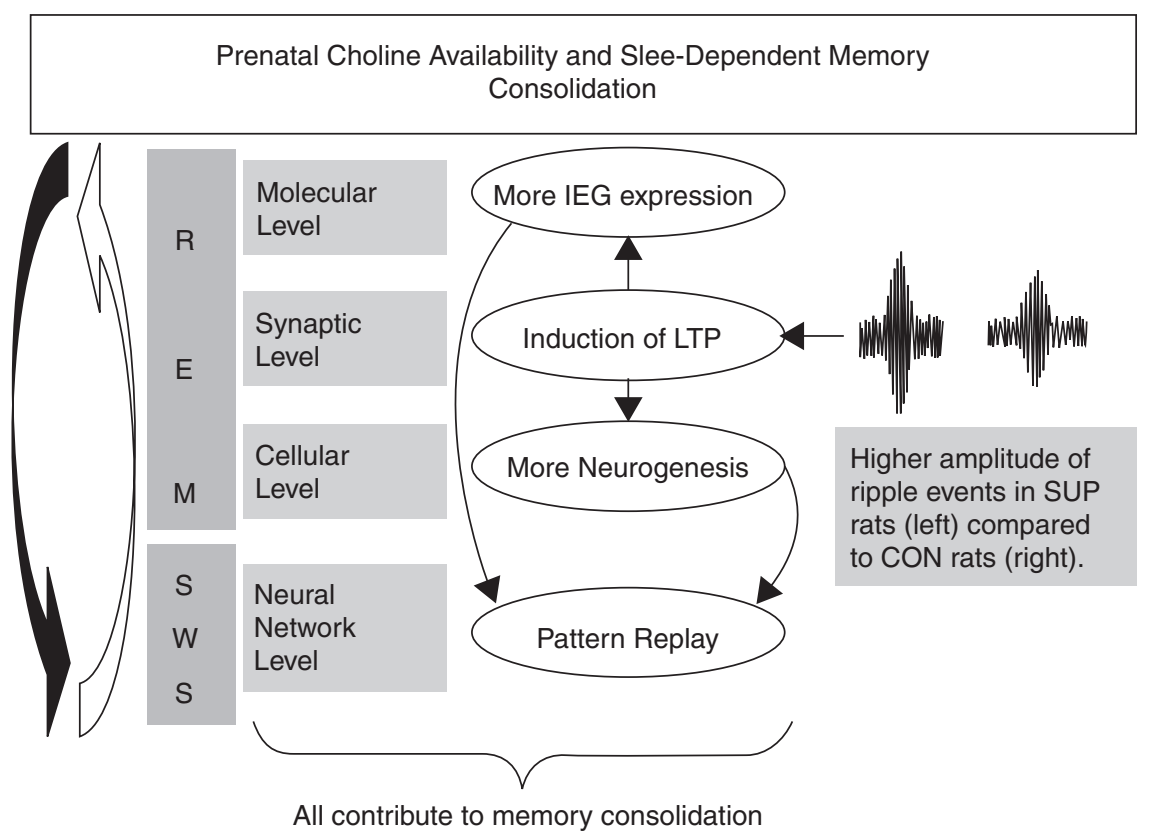

Fig. 6. Increases in the amplitude and/or frequency of hippocampal ripple events following prenatal choline supplementation contribute to what has been referred to as a "ripple effect" in adulthood. The current hypothesis is that this "ripple effect" induces differential patterns of gene expression, lowered thresholds for long-term potentiation (LTP) induction, and increases in neurogenesis. The result is that additional "raw materials" are provided during REM sleep thereby making memory consolidation during slow-wave sleep (SWS) more efficient. This proposal is supported by the observation that rats treated with prenatal choline supplementation exhibit increases in memory capacity and precision in adulthood (see 87,93 ).

memory by being utilized in the process of neuronal pattern replay and information transfer during SWS at the neural-network level. Among these neurophysiological features during sleep, hippocampal ripple event seems to play critical roles because it serves as the in vivo high-frequency stimulation that could trigger LTP and then initiate other neurophysiological changes mentioned above. This theoretical framework is supported by our findings that prenatal choline supplementation increases the amplitude of hippocampal ripple events and the occurrence of ripple events during REM sleep. The stronger hippocampal ripple events during sleep create a "ripple effect" in the brain that together lead to larger capacity, better precision, and more enduring memory in rats treated with prenatal choline supplementation. These effects also extend into old age and inoculate subjects against age-related declines in memory that may be related to disruptions in sleep in untreated individuals (46, 90, 93).

\section{References}

1. Aimone, J.B., Wiles, J. and Gage, F.H. Potential role for adult neurogenesis in the encoding of time in new memories. Nature Neurosci. 9: 723-727, 2006.

2. Aserinsky, E. and Kleitman, N. Regularly occurring periods of eye motility and concomitant phenomena, during sleep. Science 118: 273-274, 1953.
3. Axmacher, N., Elger, C.E. and Fell, J. Ripples in the medial temporal lobe are relevant for human memory consolidation. Brain 131: 1806-1817, 2008.

4. Axmacher, N., Draguhn, A., Elger, C.E. and Fell, J. Memory processes during sleep: Beyond the standard consolidation theory. Cell. Mol. Life Sci. DOI 10.1007/s00018-009-0019-1, 2009.

5. Axmacher, N., Haupt, S., Fernandez, G., Elger, C.E. and Fell, J. The role of sleep in declarative memory consolidation - direct evidence by intracranial EEG. Cereb. Cortex 18: 500-507, 2008.

6. Axmacher, N., Mormann, F., Fernandez, G., Elger, C.E. and Fell, J. Memory formation by neuronal synchronization. Brain Res. Rev. 52: 170-182, 2006.

7. Battaglia, F.P., Sutherland, G.R. and McNaughton, B.L. Hippocampal sharp wave bursts coincide with neocortical "up-state" transitions. Learn. Mem. 11: 697-704, 2004.

8. Behrens, C.J., van den Boom, L.P., de Hoz, L., Friedman, A. and Heinemann, U. Induction of sharp wave-ripple complexes in vitro and reorganization of hippocampal networks. Nat. Neurosci. 8: 1560-1567, 2005

9. Best, J., Behn, C.D., Poe, G.R. and Booth, V. Neuronal models for sleep-wake regulation and synaptic reorganization in the sleeping hippocampus. J. Biol. Rhythms 22: 220-232, 2007.

10. Bialek, W., Rieke, F., de Ruyter van Steveninck, R. R. and Warland, D. Reading a neural code. Science 252: 1854-1857, 1991.

11. Blusztajn, J.K., Cermak, J.M., Holler, T. and Jackson, D.A. Imprinting of hippocampal metabolism of choline by its availability during gestation: implications for cholinergic neurotransmission. J. Physiol. 92: 199-203, 1998.

12. Bruel-Jungerman, E., Laroche, S. and Rampon, C. New neurons in the dentate gyrus are involved in the expression of enhanced long-term memory following environmental enrichment. Eur. J. Neurosci. 21: 513-521, 2005.

13. Buzsaki, G. Hippocampal sharp waves: Their origin and significance. Brain Res. 398: 242-252, 1986. 
14. Buzsáki, G. Memory consolidation during sleep: A neurophysiological perspective. J. Sleep Res. 7: 17-23, 1998.

15. Buzsáki, G. and Draguhn, A. Neuronal oscillations in cortical networks. Science 304: 1926-1929, 2004.

16. Calamandrei, G., Ricceri, L. and Valanzano, A. Systemic administration of anti-NGF antibodies to neonatal mice impairs 24-h retention of an inhibitory avoidance task while increasing ChAT immunoreactivity in the medial septum. Behav. Brain Res. 78: 81-91, 1996.

17. Campbell, I.G., Guinan, M.J. and Horowitz, J.M. Sleep deprivation impairs long-term potentiation in rat hippocampal slices. $J$. Neurophysio. 88: 1073-1076, 2002.

18. Carskadon, M.A. and Dement, W.C. Normal human sleep: An overview. In: Kryher, M.H., Roth, T., Dement, W.C. (Eds.), Principles and practice of sleep medicine. Elsevier Saunders, Philadelphia, PA., 2005.

19. Cermak, J.M., Holler, T., Jackson, D.A. and Blusztajn, J.K. Prenatal availability of choline modifies development of the hippocampal cholinergic system. FASEB J. 12: 349-357, 1998.

20. Cermak, J.M., Blusztajn, J.K., Meck, W.H., Williams, C.L., Fitzgerald, C., Rosene, D.L. and Loy, R. Prenatal availability of choline alters the development of acetylcholinesterase in rat hippocampus. Dev. Neurosci. 21: 94-104, 1999.

21. Cheng, R.K. and Meck, W.H. Prenatal choline supplementation increases sensitivity to time by reducing non-scalar sources of variance in adult temporal processing. Brain Res. 1186: 242-254, 2007.

22. Cheng, R.K., Meck, W.H. and Williams, C.L. $\alpha 7$ nicotinic acetylcholine receptors and temporal memory: Synergistic effects of combining prenatal choline and nicotine on reinforcement-induced resetting of an interval clock. Learn. Mem. 13: 127-134, 2006.

23. Cheng, R.K., Williams, C.L. and Meck, W.H. Oscillatory bands, neuronal synchrony, and hippocampal function: Implications of the effects of prenatal choline supplementation for sleep-dependent memory consolidation. Brain Res. 1237: 176-194, 2008.

24. Cheng, R.K., Williams, C.L. and Meck, W.H. Prenatal choline supplementation enhances hippocampal gamma oscillations and increases sharp-wave ripple activity during rapid eye movement (REM) sleep following exploration of novel objects. submitted, 2009.

25. Cheng, R.K., MacDonald, C.J., Williams, C.L. and Meck, W.H Prenatal choline supplementation alters the timing, emotion, and memory performance (TEMP) of adult male and female rats as indexed by differential reinforcement of low-rate schedule behavior. Learn. Mem. 15: 153-162, 2008.

26. Cheng, R.K., Scott, A.C., Penney, T.B., Williams, C.L. and Meck, W.H. Prenatal-choline supplementation differentially modulates timing of auditory and visual stimuli in aged rats. Brain Res. 1237: 167-175, 2008.

27. Cheng, S. and Frank, L.M. New experiences enhance coordinated neural activity in the hippocampus. Neuron 57: 303-313, 2008.

28. Chklovskii, D.B., Mel, B.W. and Svoboda, K. Cortical rewiring and information storage. Nature 431: 782-788, 2004.

29. Chun, S.K., Sun, W., Park, J.J. and Jung, M.W. Enhanced proliferation of progenitor cells following long-term potentiation induction in the rat dentate gyrus. Neurobiol. Learn. Mem. 86: 322-329, 2006.

30. Clemens, Z., Molle, M., Eross, L., Barsi, P., Halasz, P. and Born, J. Temporal coupling of parahippocampal ripples, sleep spindles, and slow oscillations in humans. Brain 130: 2868-2878, 2007.

31. Cole, A.J., Saffen, D.W., Baraban, J.M. and Worley, P.F. Rapid increase of an immediate early gene messenger RNA in hippocampal neurons by synaptic NMDA receptor activation. Nature 340: 474-476, 1989.

32. Datta, S. and Hobson, J.A. The rat as an experimental model for sleep neurophysiology. Behav. Neurosci. 114: 1239-1244, 2000.

33. Datta, S. and Maclean, R.R. Neurobiological mechanisms for the regulation of mammalian sleep-wake behavior: Reinterpretation of historical evidence and inclusion of contemporary cellular and molecular evidence. Neurosci. Niobehav. Rev. 31: 775-824, 2007.

34. Datta, S., Li, G. and Auerbach, S. Activation of phasic pontinewave generator in the rat: a mechanism for expression of plasticityrelated genes and proteins in the dorsal hippocampus and amygdala. Eur. J. Neurosci. 27: 1876-1892, 2008.

35. Datta, S., Mavanji, V., Ulloor, J. and Patterson, E.H. Activation of phasic pontine-wave generator prevents rapid eye movement sleep deprivation-induced learning impairment in the rat: A mechanism for sleep-dependent plasticity. J. Neurosci. 24: 1416-1427, 2004.

36. Dement, W.C. History of sleep medicine. Neurol. Clin. 23: 945965, 2005.

37. Dement, W.C. and Kleitman, N. Cyclic variations in EEG during sleep and their relation to eye movements, body motility and dreaming. Electroencephalogr. Clin. Neurophysiol. 20: 673-690, 1957.

38. Dzirasa, K., Ribeiro, S., Costa, R., Santos, L.M., Lin, S.C., Grosmark, A., Sotnikova, T.D., Gainetdinov, R.R., Caron, M.G. and Nicolelis, M.A. Dopaminergic control of sleep-wake states. J. Neurosci. 26: 10577-10589, 2006.

39. Engel, A.K., Fries, P. and Singer, W. Dynamic predictions: oscillations and synchronization in top-down processing. Nat. Rev. Neurosci. 2: 704-716, 2001

40. Eschenko, O., Ramadan, W., Mölle, M., Born, J. and Sara, S.J. Sustained increase in hippocampal sharp-wave ripple activity during slow-wave sleep after learning. Learn. Memory 15: 373377,2008

41. Fischer, W., Björklund, A., Chen, K. and Gage, F.H. (1991). NGF improves spatial memory in aged rodents as a function of age. J. Neurosci. 11, 1889-1906.

42. Fogel, S.M., Nader, R., Cote, K.A. and Smith, C.T. Sleep spindles and learning potential. Behav. Neurosci. 121: 1-10, 2007.

43. Frankland, P.W. and Bontempi, B. The organization of recent and remote memories. Nat. Rev. Neurosci. 6: 119-130, 2005.

44. Gais, S. and Born, L. Low acetylcholine during slow-wave sleep is critical for declarative memory consolidation. Proc. Natl. Acad. Sci. USA 101: 2140-2144, 2004.

45. Gais, S., Molle, N., Helms, K. and Born, J. Learning-dependent increases in sleep spindle density. J. Neurosci. 22: 6830-6834, 2002.

46. George, O., Parducz, A., Dupret, D., Kharouby, M., Le Moal, M., Piazza, P.V. and Mayo, W. Smad-dependent alterations of PPT cholinergic neurons as a pathophysiological mechanisms of age-related sleep-dependent memory impairments. Neurobiol. Aging 27: 1848-1858, 2006.

47. Girardeau, G., Benchenane, K., Herwik, S., Kisban, S., Ruther, P., Weiner, S.I., Buzsaki, G. and Zugaro, M.B. Abolishing hippocampal ripples by timed stimulations: Effects on learning. Soc. Neurosci. Abst. 690.14, 2008.

48. Glenn, M.J., Gibson, E.M., Kirby, E.D., Mellott, T.J., Blusztajn, J.K. and Williams, C.L. Prenatal choline availability modulates hippocampal neurogenesis and neurogenic responses to enriching experiences in adult female rats. Eur. J. Neurosci. 25: 2473-2482, 2007.

49. Glenn, M.J., Gibson, E.M., Kirby, E.D., Wong-Goodrich, S.J.E, Mellott, T.J., Blusztajn, J.K. and Williams, C.L. Age-related declines in exploratory behavior and markers of hippocampal plasticity are attenuated by prenatal choline supplementation in rats. Brain Res. 1237: 110-123, 2008.

50. Gold, P.E. Protein synthesis inhibition and memory: Formation vs. amnesia. Neurobiol. Learn. Mem. 89: 201-211, 2008.

51. Gottesmann, C. Detection of seven sleep-waking stages in the rat. Neurosci. Biobehav. Rev. 16: 31-38, 1992.

52. Graves, L.A., Heller, E.A., Pack, A.I. and Abel, T. Sleep deprivation selectively impairs memory consolidation for contextual fear 
conditioning. Learn. Mem. 10: 168-176, 2003.

53. Guzman-Marin, R., Suntsova, N., Bashir, T., Neinhuis, R., Szymusiak, R. and McGinty, D. Rapid eye movement sleep deprivation contributes to reduction of neurogenesis in the hippocampal dentate gyrus of the adult rat. Sleep 31: 167-175, 2008.

54. Guzman-Marin, R., Suntsova, N., Methippara, M., Greiffenstein, R., Szymusiak, R. and McGinty, D. Sleep deprivation suppresses neurogenesis in the adult hippocampus of rats. Eur. J. Neurosci. 22: 2111-2116, 2005.

55. Guzman-Marin, R., Suntsova, N., Stewart, D.R., Gong, H., Szymusiak, R. and McGinty, D. Sleep deprivation reduces proliferation of cells in the dentate gyrus of the hippocampus in rats. J. Physiol. 542: 563-571, 2003.

56. Hairston, I.S., Little, M.T.M., Scanlon, M.D., Barakat, M.T., Palmer, T.D., Sapolsky, R.M. and Heller, G.C. Sleep restriction suppresses neurogenesis induced by hippocampus-dependent learning. J. Neurophysiol. 94: 4224-4233, 2005.

57. Huber, R., Ghilardi, M.F., Massimini, M. and Tononi, G. Local sleep and learning. Nature 430: 78-81, 2004.

58. Huerta, P.T. and Lisman, J.E. Bidirectional synaptic plasticity induced by a single burst during cholinergic theta oscillation in CA1 in vitro. Neuron 15: 1053-1063, 1995.

59. Huerta, P.T. and Lisman, J.E. Synaptic plasticity during the cholinergic theta-frequency oscillation in vitro. Hippocampus 6: 58-61, 1996.

60. Ishigawa, A., Kanayama, Y., Matsumura, H., Tsuchimochi, H., Ishida, Y. and Nakamura, S. Selective rapid eye movement sleep deprivation impairs the maintenance of long-term potentiation in the rat hippocampus. Eur. J. Neurosci. 24: 242-248, 2006.

61. Jarosiewicz, B., McNaughton, B.L. and Skaggs, W.E. Hippocampal population activity during the small-amplitude irregular activity state in the rat. J. Neurosci. 22: 1373-1384, 2002.

62. Ji, D. and Wilson, M.A. Coordinated memory replay in the visual cortex and hippocampus during sleep. Nat. Neurosci. 10: 100-107, 2007.

63. Jones III, J.P., Meck, W.H., Williams, C.L., Wilson, W.A. and Swartzwelder, S.H. Choline availability to the developing rat fetus alters adult hippocampal long-term potentiation. Dev. Brain Res. 118: 159-167, 1999.

64. Jones, M.W. and Wilson, M.A. Theta rhythms coordinate hippocampal-prefrontal interactions in a spatial memory task. PLoS Bio. 3: 2187-2199, 2005.

65. Káli, S. and Dayan, P. Off-line replay maintains declarative memories in a model of hippocampal-neocortical interactions. Nat. Neurosci. 7: 286-294, 2004.

66. Kandel, E.R. The molecular biology of memory storage: A dialogue between genes and synapses. Science 294: 1030-1038, 201.

67. Kee, N., Teixeira, C.M., Wang, A.G. and Frankland, P.W. Preferential incorporation of adult-generated granule cells into spatial memory networks in the dentate gyrus. Nat. Neurosci. 10: 355-362, 2007.

68. Kudrimoti, H.S., Barnes, C.A. and McNaughton, B.L. Reactivation of hippocampal cell assemblies: Effects of behavioral state, experience, and EEG dynamics. J. Neurosci. 19: 4090-4101, 1999.

69. Le Van Quyen, M., Bragin, A., Staba, R., Crepon, B., Wilson, C.L. and Engel, J. Jr. Cell type-specific firing during ripple oscillations in the hippocampal formation of humans. J. Neurosci. 28: 6104-6110, 2008.

70. Lee, A.K. and Wilson, M.A. Memory of sequential experience in the hippocampus during slow wave sleep. Neuron 36: 1183 1194, 2002.

71. Li, Q., Guo-Ross, S., Lewis, D.V., Turner, D., White, A.M., Wilson, W.A. and Swartzwelder, H.S. Dietary prenatal choline supplementation alters postnatal hippocampal structure and function. $J$. Neurophysiol. 91: 1545-1555, 2004.

72. Lopez, J., Roffwarg, H.P., Dreher, A., Bissette, G., Karolewicz, B. and Shaffery, J.P. Rapid eye movement sleep deprivation decreases long-term potentiation stability and affects some glutamatergic signaling proteins during hippocampal development. Neurosci. 153: 44-53, 2008.

73. Louie, K. and Wilson, M.A. Temporally structured replay of awake hippocampal ensemble activity during rapid eye movement sleep. Neuron 29: 145-146, 2001.

74. Lyons, R.G. Understanding digital signal processing. 2nd Edition, Prentice Hall, Upper Saddle River, NJ, 2004.

75. Mahon, S., Vautrelle, N., Pezard, L., Slaght, S.J., Deniau, J.M., Chouvet, G. and Charpier, S. Distinct patterns of striatal medium spiny neuron activity during the natural sleep-wave cycle. $J$. Neurosci. 26: 12587-12595, 2006.

76. Maier, N. Güldenagel, M., Söhl, G., Siegmund, H., Willecke, K. and Draguhn, A. Reduction of high-frequency network oscillations (ripples) and pathological network discharges in hippocampal slices from connexin 36-deificent mice. J. Physiol. 541: 521-528, 2002.

77. Marshall, L. and Born, J. The contribution of sleep to hippocampusdependent memory consolidation. Trends Cogn. Sci. 11: 442-450, 2007.

78. Marshall, L., Helgadottir, H., Mölle, M. and Born, J. Boosting slow oscillations during sleep potentiates memory. Nature 444: 610-613, 2006.

79. McClelland, J.L., McNaughton, B.L. and O'Reilly, R.C. Why there are complementary learning systems in the hippocampus and neocortex: Insights from the successes and failures of connectionist models of learning and memory. Psychol. Rev. 102: 419-457, 1995.

80. McGowan, P.O., Meaney, M.J. and Szyf, M. Diet and the epigenetic (re)programming of phenotypic differences in behavior. Brain Res. 1237: 12-24, 2008.

81. Meck, W.H. Choline uptake in the frontal cortex is proportional to the absolute error of a temporal memory translation constant in mature and aged rats. Learn. Motiv. 33: 88-104, 2002.

82. Meck, W.H. Hippocampal function is required for feedback control of an internal clock's criterion. Behav. Neurosci. 102: 54-60, 1988.

83. Meck, W.H. Neuropharmacology of timing and time perception. Cog. Brain Res. 3: 227-242, 1996.

84. Meck, W.H., Church, R.M. and Olton, D.S. Hippocampus, time, and memory. Behav. Neurosci. 98: 3-22, 1984.

85. Meck, W.H. and Williams, C.L. Characterization of the facilitative effects of perinatal choline supplementation on timing and temporal memory. Neuroreport 8: 2831-2835, 1997.

86. Meck, W.H. and Williams, C.L. Choline supplementation during pre- and postnatal development reduces proactive interference in spatial memory. Dev. Brain Res. 118: 51-59, 1999.

87. Meck, W.H. and Williams, C.L. Metabolic imprinting of choline by its availability during gestation: Implications for memory and attentional processing across the lifespan. Neurosci. Biobehav. Rev. 27: 385-399, 2003.

88. Meck, W.H. and Williams, C.L. Prenatal choline supplementation increases the threshold for chunking in spatial memory. Neuroreport 8: 3053-3059, 1997.

89. Meck, W.H. and Williams, C.L. Simultaneous temporal processing is sensitive to prenatal choline availability in mature and aged rats. Neuroreport 8: 3045-3051, 1997.

90. Meck, W.H., Church, R.M. and Wenk, G. L. Arginine vasopressin inoculates against age-related increases in sodium-dependent high affinity choline uptake and discrepancies in the content of temporal memory. Eur. J. Pharmacol. 130: 327-331, 1986.

91. Meck, W.H., Smith, R.A. and Williams, C.L. Organizational changes in cholinergic activity and enhanced visuospatial memory as a function of choline administered prenatally or postnatally or both. Behav. Neurosci. 103: 1234-1241, 1989.

92. Meck, W.H., Smith, R.A. and Williams, C.L. Pre- and postnatal choline supplementation produces long-term facilitation of spatial memory. Dev. Psychobiol. 21: 339-353, 1988.

93. Meck, W.H., Williams, C.L., Cermak, J.M. and Blusztajn, J.K. Developmental periods of choline sensitivity provide an ontoge- 
netic mechanism for regulating memory capacity and age-related dementia. Front. Integr. Neurosci., doi:10.3389/neuro.07.007. 2007 1:7, 2008.

94. Melgire, M., Ragot, R., Samson, S., Penney, T.B., Meck, W.H. and Pouthas, V. Auditory/visual duration bisection in patients with left or right medial-temporal lobe resection. Brain Cog. 58: 119-124, 2005.

95. Mellott, T.J., Williams, C.L., Meck, W.H. and Blusztajn, J.K. Prenatal choline supplementation advances hippocampal development and enhances MAPK and CREB activation. FASEB J. 18: 545-547, 2004.

96. Mellott, T.J., Follettie, M.T., Diesl, V., Hill, A.A., Lopez-Coviella, I. and Blusztajn, J.K. Prenatal choline availability modulates hippocampal and cerebral cortical gene expression. FASEB $J$. 21: 1311-132, 2007.

97. Mohler, E.G., Meck, W.H. and Williams, C.L. Sustained attention in adult mice is modulated by prenatal choline availability. Inter. J. Comp. Psychol. 14: 136-150, 2001.

98. Mölle, M., Marshall, L., Gais, S. and Born, J. Grouping of spindle activity during slow oscillations in human non-rapid eye movement sleep. J. Neurosci. 22: 10941-10947, 2002.

99. Mölle, M., Yeshenko, O., Marshall, L., Sara, S.J. and Born, J. Hippocampal sharp wave-ripples linked to slow oscillations in rat slow-wave sleep. J. Neurophysiol. 96: 62-70, 2006.

100. Montoya, D.A., White, A.M., Williams, C.L., Blusztajn, J.K., Meck, W.H. and Swartzwelder, H.S. Prenatal choline exposure alters hippocampal responsiveness to cholinergic stimulation in adulthood. Dev. Brain Res. 123: 25-32, 2000.

101. Murphy, M., Riedner, B.A., Huber, R., Massimini, M., Ferrarelli, F. and Tononi, G. Source modeling sleep slow waves. Proc. Natl. Acad. Sci. USA 106: 1608-1613, 2009.

102. Nishida, M., Pearsall, J., Buckner, R.L. and Walker, M.P. REM sleep, prefrontal theta, and the consolidation of human emotional memory. Cereb. Cortex 19: 1158-1166, 2009.

103. Papatheodoropoulos, C. A possible role of ectopic action potentials in the in vitro hippocampal sharp wave-ripple complexes. Neurosci. 157: 495-501, 2008.

104. Pavlides, C., Greenstein, Y.J., Grudman, M. and Winson, J. Longterm potentiation in the dentate gyrus is induced preferentially on the positive phase of theta-rhythm. Brain Res. 439: 383-387, 1988.

105. Pennartz, C.M., Lee, E., Verheul, J., Lipa, P., Barnes, C.A. and McNaughton, B.L. The ventral striatum in off-line processing: Ensemble reactivation during sleep and modulation by hippocampal ripples. J. Neurosci. 24: 6446-6456, 2004.

106. Planke, D.T. and Winkelman, J.W. Sleep disturbance in bipolar disorder: Therapeutic implications. Am. J. Psychiatry 165: 830843, 2008.

107. Pogosyan, A., Kuhn, A., Trottengerg, T., Schneider, G.-H., Kupsch, A. and Brown, P. Elevations in local gamma activity are accompanied by changes in the firing rate and information coding capacity of neurons in the regions of the subthalamic nucleus in Parkinson's disease. Exp. Neurol. 202: 271-279, 2006.

108. Ponomarenko, A.A., Li, J.S., Korotjova, T.M., Huston, J.P. and Haas, H.L. Frequency of network synchronization in the hippocampus marks learning. Eur. J. Neurosci. 27: 3035-3042, 2008.

109. Power, A.E. Slow-wave sleep, acetylcholine, and memory consolidation. Proc. Natl. Acad. Sci. USA 101: 1795-1796, 2004.

110. Pyapali, G.K., Turner, D.A., Williams, C.L., Meck, W.H. and Swartzwelder, H.S. Prenatal dietary choline supplementation decreases the threshold for induction of long-term potentiation in young adult rats. J. Neurophysiol. 794: 238-255, 1998.

111. Qiang, L., Guo-Ross, S., Lewis, D.V., Turner, D., White, A.M., Wilson, W.A. and Swartzwelder, H.S.J. Dietary prenatal choline supplementation alters postnatal hippocampal structure and function. Neurophysiol. 91: 1545-1555, 2004.

112. Rasch, B., Pommer, J., Diekelmann, S. and Born, J. Pharmacological REM sleep suppression paradoxically improves rather than impairs skill memory. Nat. Neurosci. 12: 396-397, 2009.

113. Ribeiro, S., Goyal, V., Mello, C.V. and Constantine, P. Brain gene expression during REM sleep depends on prior waking experience. Learn. Mem. 6: 500-508, 1999.

114. Ribeiro, S., Mello, C.V., Velho, T., Gardner, T.J., Jarvis, E.D. and Pavlides, C. Induction of hippocampal long-term potentiation during waking leads to increased extrahippocampal zif-268 expression during ensuing rapid-eye-movement sleep. J. Neurosci. 22: 10914-10923, 2002.

115. Robert, C., Guilpin, C. and Limoge, A. Automated sleep staging systems in rats. J. Neurosci. Methods 88: 111-122, 1999.

116. Robertson, E.M. From creation to consolidation: A novel framework for memory processing. PLoS Biol. 7: e19, 2009.

117. Sandstrom, N.J., Loy, R. and Williams, C.L. Prenatal choline supplementation increases NGF levels in the hippocampus and frontal cortex of young and adult rats. Brain Res. 947: 9-16, 2002.

118. Sejnowski, T.J. and Destexje, A. Why do we sleep? Brain Res. 886: 208-223, 2000.

119. Shimizu, E., Tang, Y.P., Rampon, C. and Tsien, J.Z. NMDA receptor-dependent synaptic reinforcement as a crucial process for memory consolidation. Science 290: 1170-1174, 2000.

120. Shors, T.J. From stem cells to grandmother cells: How neurogenesis relates to learning and memory. Cell Stem Cell 11: 253-258, 2008.

121. Siapas, A.G. and Wilson, M.A. Coordinated interactions between hippocampal ripples and cortical spindles during slow-wave sleep. Neuron 21: 1123-1128, 1998.

122. Siegel, J.M. The REM sleep-memory consolidation hypothesis. Science 294: 1058-1063, 2001.

123. Sirota, A., Csicsvari, J., Buhl, D. and Buzsáki, G. Communication between neocortex and hippocampus during sleep in rodents. Proc. Natl. Acad. Sci. USA 100: 2065-2069, 2003.

124. Skaggs, W.E. and McNaughton, B.L. Replay of neuronal firing sequence in rat hippocampus during sleep following spatial experience. Science 271: 1870-1873, 1996.

125. Skaggs, W.E., McNaughton, B.L., Permenter, M., Archibeque, M., Vogt, J., Amaral, D.G. and Barnes, C.A. EEG sharp waves and sparse ensemble unit activity in the macaque hippocampus. J. Neurophysiol. 98: 898-910, 2007.

126. Smith, C. and Rose, G.M. Evidence for a paradoxical sleep window for place learning in the Morris water maze. Physiol. Behav. 59: 93-97, 1996.

127. Smith, C. and Rose, G.M. Posttraining paradoxical sleep in rats is increased after spatial learning in the Morris water maze. Behav. Neurosci. 111: 1197-1204, 1997.

128. Smith, C. and Wong, P.T.P. Paradoxical sleep increases predict successful learning in a complex operant task. Behav. Neurosci. 105: 282-288, 1991.

129. Smith, C.T., Nixon, M.R. and Nader, R.S. Posttraining increases in REM sleep intensity implicate REM sleep in memory processing and provide a biological marker of learning potential. Learn. Mem. 11: 714-719, 2004.

130. Spencer, R.M., Sunm, M. and Ivry, R.B. Sleep-dependent consolidation of contextual learning. Curr. Biol. 16: 1001-1005, 2006.

131. Steriade, M. Brain electrical activity and sensory processing during waking and sleep states. In: Kryher, M.H., Roth, T., Dement, W.C. (Eds.), Principles and practice of sleep medicine. Elsevier Saunders, Philadelphia, PA, pp. 101-119, 2005.

132. Steriade, M. and Amzica, F. Intracortical and corticothalamic coherency of fast spontaneous oscillations. Proc. Natl. Acad. Sci. USA 93: 2533-2538, 1996.

133. Steriade, M. and Timofeev, I. Neuronal plasticity in thalamocortical networks during sleep and waking oscillations. Neuron 37: 563-576, 2003.

134. Steriade, M., Amzica, F. and Contreras, D. Synchronization of fast $(30-40 \mathrm{~Hz})$ spontaneous cortical rhythms during brain activation. J. Neurosci. 16: 392-417, 1996. 
135. Steriade, M., Timofeev, I. and Grenier, F. Natural waking and sleep states: a view from inside neocortical neurons. J. Neurophysiol. 85: 1969-1985, 2001.

136. Stickgold, R. and Walker, M.P. Memory consolidation and reconsolidation: What is the role of sleep? Trends Neurosci. 28: 408-415, 2005.

137. Takehara-Nishiuchi, K., Nakao, K., Kawahara, S., Matsuki, N. and Kirino, Y. Systems consolidation requires postlearning activation of NMDA receptors in the medial prefrontal cortex in trace eyeblink conditioning. J. Neurosci. 26: 5049-5058, 2006.

138. Tucker, M.A. and Fishbein, W. Enhancement of declarative memory performance following a daytime nap is contingent on strength of initial task acquisition. Sleep 31: 197-203, 2008.

139. Tyzio, R., Repressa, A., Jorquera, I., Ben-Ari, Y., Gozlan, H. and Aniksztejn, L. The establishment of GABAergic and glutamatergic synapses on CA1 pyramidal neurons is sequential and correlates with the development of the apical dendrite. J. Neurosci. 19: 1037210382, 1999.

140. Varela, F., Lachaux, J.-P., Rodriguez, E. and Martinerie, J. The brainweb: Phase synchronization and large-scale integration. Nat. Rev. Neurosci. 2: 229-239, 2001.

141. van Praag, H., Schinder, A.F., Christie, B.R., Toni, N., Palmer, T.D. and Gage, F.H. Functional neurogenesis in the adult hippocampus. Nature 415: 1030-1034, 2002.

142. Wagner, U. and Born, J. Memory consolidation during sleep; interactive effects of sleep stages and HPA regulation. Stress 11: 28-41, 2008.

143. Walker, M.P. and Stickgold, R. Sleep, memory, and plasticity. Ann. Rev. Psychol. 57: 139-166, 2006.

144. Waterland, R.A. and Jirtle, R.L. Early nutrition, epigenetic changes at transposons and imprinted genes, and enganced susceptibility to adult chronic disease. Nutrition 20: 63-68, 2004.
145. Wierzynski, C.M., Lubenov, E.V., Gu, M. and Siapas, A.G. Statedependent spike-timing relationships between hippocampal and prefrontal circuits during sleep. Neuron 61: 587-596, 2009.

146. Williams, C.L. Food for thought: Brain, genes, and nutrition. Brain Res. 1237: 1-4, 2008.

147. Williams, C.L., Meck, W.H., Heyer, D. and Loy, R. Hypertrophy of basal forebrain neurons and enhanced visouspatial memory in perinatally choline-supplemented rats. Brain Res. 794: 225238, 1998.

148. Wilson, M.A. and McNaughton, B.L. Reactivation of hippocampal ensemble memories during sleep. Science 265: 676-679, 1994.

149. Womelsdorf, T., Schoffelen, J.-M., Oostenveld, R., Singer, W., Desimone, R., Engel, A.K. and Fries, P. Modulation of neuronal interactions through neuronal synchronization. Science 316: 16091612, 2007.

150. Wong, R.O.L. and Ghosh, A. Activity-dependent regulation of dendritic growth and patterning. Nat. Rev. Neurosci. 3: 803-812, 2002.

151. Wong-Goodrich, S.J., Glenn, M.J., Mellott, T.J., Blusztajn, J.K., Meck, W.H. and Williams, C.L. Hippocampal plasticity and spatial memory are differentially sensitive to the availability of choline in adulthood as a function of its supply in utero. Brain Res. 1237: 153166,2008

152. Wong-Goodrich, S.J.E., Mellott, T.J., Glenn, M.J., Blusztajn, J.K. and Williams, C.L. Prenatal choline supplementation attenuates neuropathological response to status epilepticus in the adult rat hippocampus. Neurobiol. Dis. 30: 255-269, 2008.

153. Zeisel, S.H. Genetic polymorphisms in methyl-group metabolism and epigenetics: Lessons from humans and mouse models. Brain Res. 1237: 5-11, 2008

154. Zeisel, S.H. Epigenetic mechanisms for nutrition determinants of later health outcomes. Am. J. Clin. Nutr. 89: 1488S-1493S, 2009. 